\title{
THE EPIDEMIOLOGY OF VEGETARIANISM AND HEALTH
}

\author{
MARGARET THOROGOOD
}

Health Promotion Sciences Unit, Department of Public Health and Policy, London

School of Hygiene and Tropical Medicine, Keppel Street, London WC1E 7HT

\section{CONTENTS}

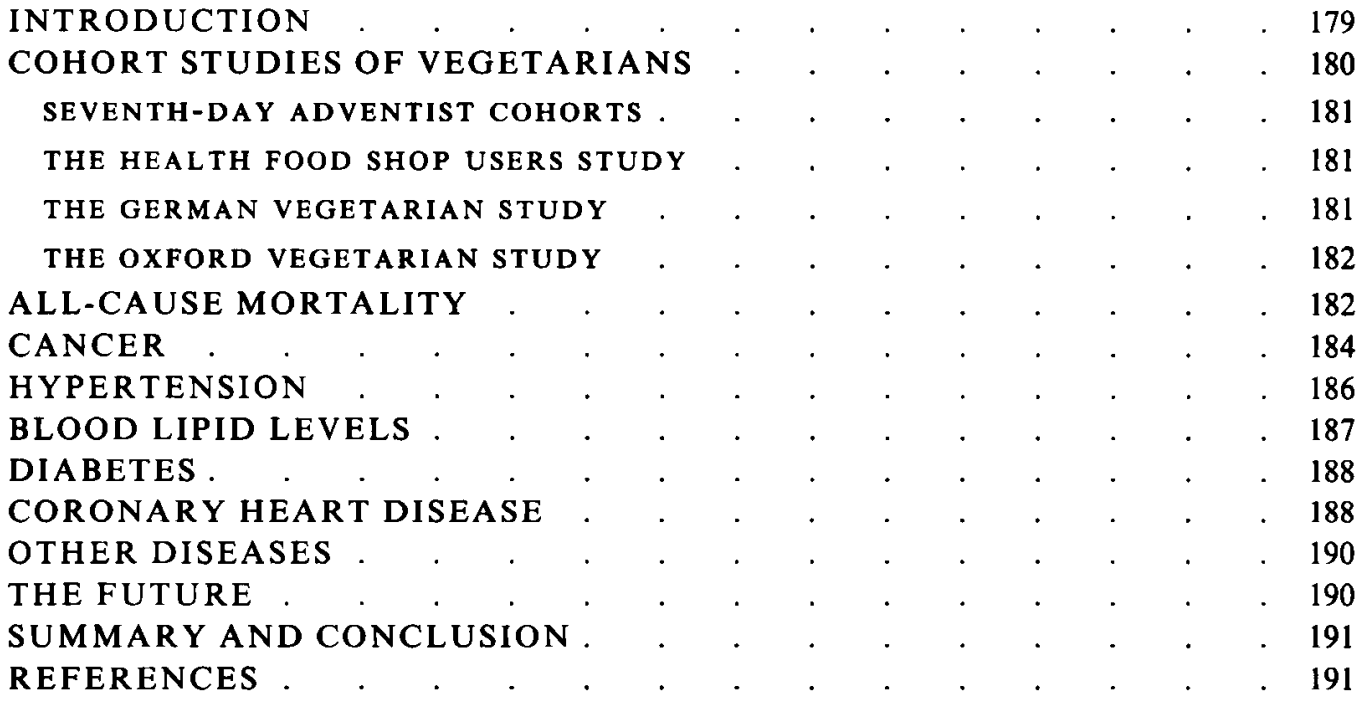

\section{INTRODUCTION}

A key problem in nutritional epidemiology is the very narrow range of dietary intakes within one population. Most of the population in the UK, for example, eat somewhere around $40 \%$ of their calories as fat and so attempts to study relationships between fat intake and disease are frustrated by the lack of subjects who eat a great deal more or a great deal less fat than the general population. Vegetarians, on the other hand, do eat a diet which is markedly different, characterized not just by the absence of meat but also by increased consumption of dietary fibre, a high polyunsaturated to saturated fat ratio, and so on. This makes vegetarians attractive subjects for epidemiologic studies.

The health consequences of eating a diet which does not include meat have been studied by a few epidemiologists, notably amongst the Seventh-Day Adventist community, for many years. Now interest in the epidemiology of vegetarianism is rapidly growing as part of a wider burgeoning interest in the role of diet in determining health. It has been established that diet plays a crucial part in determining the serum lipid and blood pressure levels, and hence the cardiovascular risk, both of individuals and of populations. Meat is an important source of saturated fatty acids in the diet, and meat-containing diets might increase the risk of cardiovascular disease. Diet also appears to be an important factor in many cancers. The potential detrimental effects of oxygen radicals in living systems have 
led nutritionists and epidemiologists to a renewed interest in the role of antioxidantcontaining fresh fruit and vegetables in the diet (Diplock, 1991). Antioxidants appear to be important both in the aetiology of some cancers and in the development of atherosclerosis, where the important role of antioxidant vitamins is to limit free radical damage to lipoproteins.

Alongside the growing scientific interest in the role of diet, some parts of the developed world (particularly where there is a Protestant heritage) have seen a rapid increase in the number of people choosing a vegetarian diet, often for ideologic rather than health promoting reasons. Vegetarians are now a substantial minority in many countries other than those, such as India, where there is a longstanding tradition of vegetarianism. Estimates of the prevalence of vegetarianism vary, but it is likely that around $4-5 \%$ of the British population are currently vegetarian. Results from a survey of three and half thousand people carried out in 1991 on behalf of the Vegetarian Society indicated that around 3.6 million people (approximately 7\% of the population) were vegetarian (Vegetarian Society, 1991), while a Gallup Poll in 1993 suggested that $4.3 \%$ of the population were vegetarians, which is double the $2 \cdot 1 \%$ observed by a similar poll in 1984 (Realeat, 1984, 1993).

Taken together, the increasing numbers of vegetarians, the possibility that such a diet protects against major causes of death, and the ecologic benefits of reduced livestock rearing (McMichael, 1992) make the public health implications of vegetarianism significant.

Vegetarianism tends to be part of a whole lifestyle, usually implying a general concern for health and for health promoting behaviour and possibly a higher resistance to seeking conventional medical treatment. The diet eaten by a vegetarian does not differ from that of his or her fellows simply in the absence of meat, but also in many other important factors such as increased consumption of vegetables, fruit and nuts. Many studies have found that vegetarians are more likely to be in non-manual jobs, tend to be slimmer, smoke less, and drink less alcohol. All of these differences may account in part for the different health experience of vegetarians. It is also possible that vegetarians have different perceptions of their state of health. A study which compared vegetarian and non-vegetarian members of the Adventist Church found significantly lower levels of self-reporting of some chronic diseases as well as significantly less use of medical services (Knutsen, 1994). The author interpreted this as showing that a vegetarian diet may decrease the prevalence of chronic disease and use of medical services. However, another interpretation might be that, for the same level of discomfort or ill health, vegetarians were less inclined to seek medical help and diagnosis. Disentangling these different effects in order to determine the importance of meat in the diet as a risk factor is a major, unsolved problem currently facing nutritional epidemiologists.

A recent important and comprehensive review of the health advantages and disadvantages of a vegetarian diet concluded that while vegetarians had a lower mortality from coronary heart disease and some forms of cancer it was impossible to determine whether a non-meat diet was conducive to good health, or whether such effects were due to other differences in the nutrients consumed by vegetarians (Dwyer, 1991). However, the review paid little attention to the relative strength of associations demonstrated in epidemiologic studies. The debate remains wide open.

\section{COHORT STUDIES OF VEGETARIANS}

Epidemiologic evidence on the health consequences of meat eating comes from a wide range of studies, including cross-sectional surveys, case-control studies and clinical trials, and this review cites some evidence from all these sources. An important part of the data 
on vegetarianism comes from a small number of cohort studies, which will be referred to repeatedly and which are described here. Cohort studies involve long term follow-up of groups of people exposed to a factor (in this case, not eating meat). The rate of events in the exposed people is compared with the rate in either a similar group of non-exposed people, or in the general population. The results are often given in terms of a standardized mortality ratio (SMR), which shows how the two rates compare after adjustment for differences in the age and sex distribution of the populations. Thus an SMR of 100 indicates that the rate is the same as that in the comparison population, lower SMR indicate a protective effect and higher SMR indicate an increased risk.

\section{SEVENTH-DAY ADVENTIST COHORTS}

Many of the cohort studies of vegetarians have been based on religious groups where meat eating is either forbidden or discouraged. One such group is the Seventh-Day Adventist Church. Adventists are instructed to avoid tobacco, alcohol, and stimulating drink (such as coffee), and are also advised by their church to follow a vegetarian diet. About half the Adventists are vegetarian, and regular meat consumption is unusual. The largest Adventist Health study involves 24044 Californian Adventists age 35 and over, followed since 1960. The study has provided a wealth of epidemiologic data on the relationship between diet and disease, and continues to generate new data on the health effects of vegetarian and semivegetarian diets. A cohort of 7285 Norwegian Adventists has also been followed since 1960 .

There are many aspects of belonging to a religious community which might affect risk of disease, such as conservative social mores, good social support and so on, and these factors may explain some of the apparent effects of the vegetarian diet. Several attempts have been made to identify groups of vegetarians who have chosen a vegetarian way of life for reasons other than membership of a religious group.

\section{THE HEALTH FOOD SHOP USERS STUDY}

A study of 10943 people with a particular interest in health foods has provided a certain amount of information on non-meat eaters. Subjects were recruited in 1973 through leaflets placed in British health food shops and through advertisements placed in vegetarian magazines, as well as by word of mouth. They completed a very simple screening questionnaire relating to the frequency of intake of high fibre foods, and were also asked whether or not they were vegetarian. Forty-three percent described themselves as vegetarian. The latest analysis available for this study relates to the period up to 1985 .

\section{THE GERMAN VEGETARIAN STUDY}

In Germany, a group of 1904 vegetarians was identified by attaching a short screening questionnaire to several vegetarian magazines. They were followed up for 11 years. Unfortunately, no comparison control cohort was followed up, so the results are difficult to interpret. Vegetarians have been shown on a number of occasions to have a lower mean body mass index, smoke fewer cigarettes and drink less alcohol, eat more fresh fruit and vegetables, and have a lower intake of saturated fatty acids and a higher intake of polyunsaturated fatty acids. All these might be important influences on mortality and it would be easier to disentangle the influence of meat avoidance if a health-aware, but meat eating, control group had also been studied. 
Table 1. Characteristics of subjects in the Oxford Vegetarian Study

\begin{tabular}{lcc}
$==$ & Meat eaters (\%) & Non-Meat eaters (\%) \\
\hline Current smokers & 24 & 16 \\
Body mass index in & & \\
1st quintile $\left(<19.9 \mathrm{~kg} / \mathrm{m}^{2}\right)$ & 13 & 25 \\
5th quintile $\left(>24.1 \mathrm{~kg} / \mathrm{m}^{2}\right)$ & 27 & 14 \\
Social class I or II & 51 & 47 \\
\hline
\end{tabular}

\section{THE OXFORD VEGETARIAN STUDY}

The Oxford Vegetarian Study cohort consists of around 6000 non-meat eaters (including around 1300 subjects who ate fish and a small group of less than 400 vegans) and 5000 meat eaters. They were recruited from all over Britain, and have been followed up since 1980 . The non-meat eaters were recruited through publicity in vegetarian journals and the national media, while the meat eaters were recruited by asking the non-meat eaters to give the names of friends and relatives. As a consequence of this method of recruitment the meat eating controls are a particularly health conscious group, and eat a moderate fat diet. All the participants have been followed up, and an analysis has been carried out on the deaths experienced up to March 1993. Because the numbers in two of the diet groups were very small, for the purposes of mortality analysis the three non-meat eating groups were analysed as one group in comparison with the meat eating group. Although an attempt was made to choose a control group which was as similar as possible to the non-meat eating group, there were still a number of minor differences between the two groups, particularly with respect to body mass and smoking. The proportion of smokers in this study overall was relatively small, but more of the meat eaters were smokers. Meat eaters were also a little heavier than the non-meat eaters, and were marginally more likely to be in social classes I or II. These differences are shown in Table 1.

\section{ALL-CAUSE MORTALITY}

Studies which have compared total mortality in vegetarians with that in the general population have found the mortality amongst the vegetarians to be around half. This can be seen in Table 2. The mainly vegetarian Californian Adventists had SMR of 53 and 63 in comparison with US white men and women respectively after 17 years of follow-up (Phillips et al. 1980). Participants in the German Vegetarian study experienced an all-cause SMR of 44 (95\% confidence interval (CI) 36-55) for men and 53 (95\% CI 44-64) for women, compared with the German population (Chang-Claude et al. 1992). Among the Health Food Shop users cohort the all-cause SMR for vegetarians was 53 (Burr \& Butland, 1988). After twelve years, the all-cause SMR for non-meat eaters in the Oxford Vegetarian Study was 41 (95\% CI 35-46) (Thorogood et al. 1994).

These findings are strongly affected by the choice of comparison group. For example, when the Californian Adventists were compared with a selected group of non-Adventist non-smokers, the size of the reduction in risk was much smaller, although the Adventists still had a significantly lower risk (Phillips, 1975; Phillips et al. 1978). The Norwegian Adventist study (Fonnebo, 1994) found a much smaller decrease in all-cause SMR in comparison with the Norwegian population; the SMR for male Adventists was 82 (95\% CI 77-88), while for women it was 95 (91-100). These smaller differences, which were 
Table 2. Standardized mortality ratios for all-cause mortality

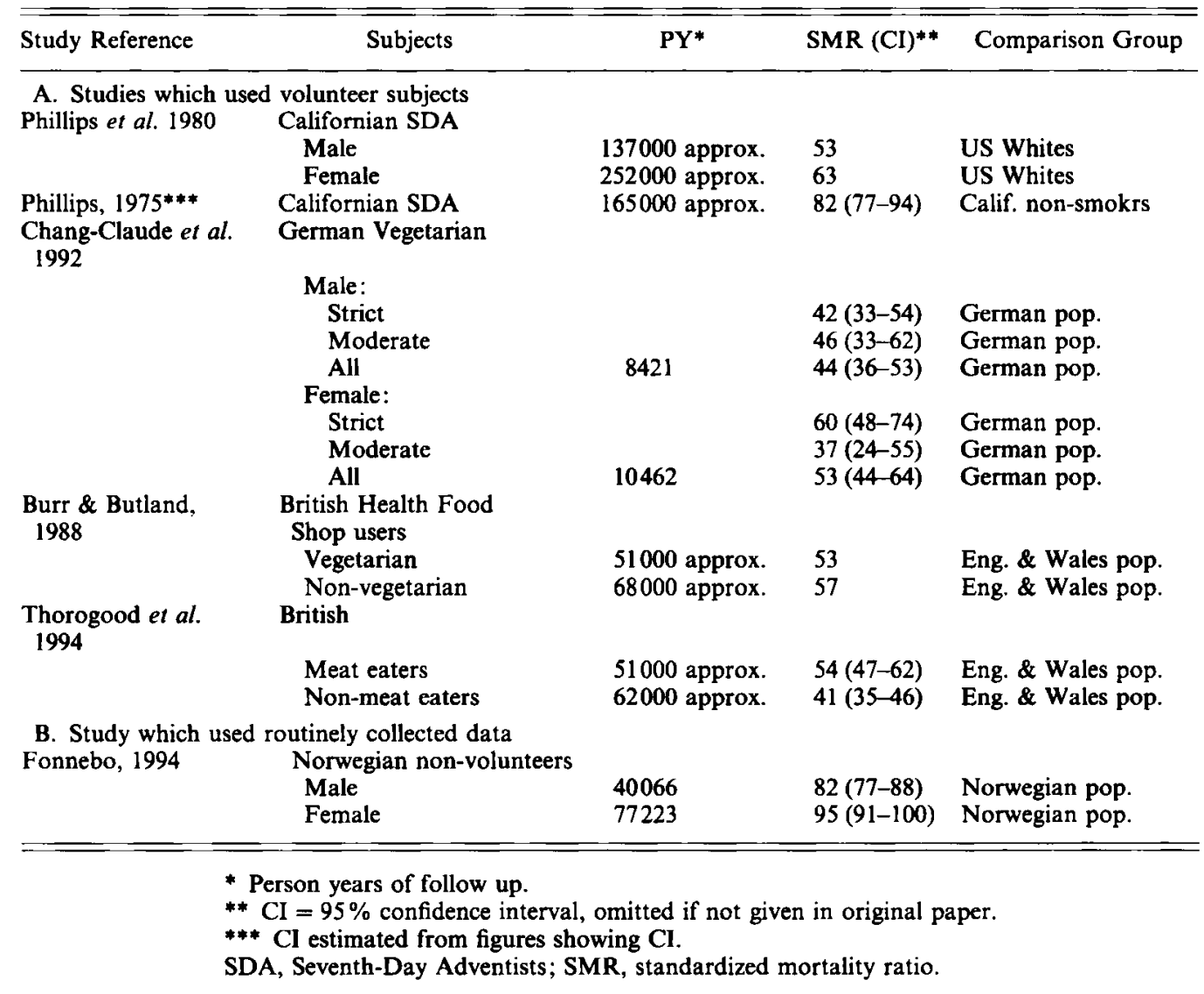

significant only in the men, may help to explain some of the differences observed in other studies. The authors note that the Adventist cohort they followed, unlike the Californian one, was not recruited from volunteers, but was identified from routinely collected data. It is therefore possible that at least some of the health advantage seen in other studies is due to the 'healthy volunteer effect', in that people who volunteer to take part in studies almost always enjoy better health than those who do not.

Comparisons within cohort studies provide conflicting data. In the Oxford Vegetarian study, the meat eaters also had a low SMR of 54 (95\% CI 47-62). The two groups (meat eaters and non-meat eaters) differed a little in other risk factors such as smoking, social class and body mass index. After adjustment had been made for these confounding factors, the relative risk of death in the non-meat eaters compared to meat eaters was $0.80(95 \%$ CI 0.65-0.99): even though the meat eaters have a beneficial death rate, the non-meat eaters had only $80 \%$ of the mortality risk of the comparison group of (very healthy) meat eaters (Thorogood et al. 1994). However, subjects in the Health Food Shop Users study who did not report themselves to be vegetarian enjoyed a low death rate (SMR 57), which was not significantly greater than that in the vegetarians (Burr \& Butland, 1988).

A re-analysis of the data from the Californian Adventist Study showed that meat consumption had a moderate but significant association with all-cause mortality in males, such that the relative risk for men eating meat 6 times a week was about $50 \%$ greater than 
that in men who ate no meat. However, there was no relationship at all between meat eating and all-cause mortality in females.

In the German Vegetarian study, the data were re-analysed with the cohort divided according to their answers to the initial questionnaire into two groups: 'strict vegetarians' who never ate meat or fish, and 'moderate vegetarians' who would eat meat or fish occasionally ('occasionally' was not defined). Mortality in the two subgroups was compared, and the moderate group was found to have a lower rate of total mortality, although the difference was not statistically significant. The relative risk of death was 0.83 $(95 \% \mathrm{Cl} 0 \cdot 62-1 \cdot 11)$ in the moderate group as compared to the strict group. The authors commented that this difference may be due to the nutritional inadequacy of the strict vegetarian diet, and pointed to the four deaths from anaemia experienced in the strict group, compared with no such deaths in the moderate group (Chang-Claude et al. 1993). It is important, however, not to place too much emphasis on a difference which was not statistically significant.

The data reported here are equivocal. Some results suggest that the avoidance of meat is related to the low mortality observed, while others indicate that it is not meat eating, but other aspects of the cohorts, which are related to the low mortality. There are several different factors to take into consideration. Apart from the Norwegian Adventist study, all the cohorts are based on volunteers and are therefore susceptible to a 'healthy volunteer' effect. The lifestyle of vegetarians may differ from that of comparison groups in other health-giving ways, including not just the important influence of tobacco use but also, for example, attitudes to exercise. Finally, even if it is the diet which accounts for the lower mortality, we cannot be certain that it is the avoidance of meat which is having an effect, rather than other aspects of diet, for example, the consumption of large quantities of vegetables, which is closely associated with not eating meat.

\section{CANCER}

Table 3 shows the standardized mortality and/or incidence ratios for cancer in the various cohorts. The Oxford Vegetarian Study reported a significant $40 \%$ reduction in death from cancer amongst the non-meat eaters compared with meat eaters (Thorogood et al. 1994). The cancer death rate ratio comparing deaths in non-meat eaters to meat eaters adjusted for body mass index, social class and cigarette smoking was 0.61 (95\% CI 0.44-0.84). Unfortunately, there were too few cases of cancer for the relative risks of death for individual cancer sites to be calculated.

Other studies have found conflicting results. The Norwegian Adventist study (Fonnebo, 1994) did not observe any lower incidence of cancer in the cohort (standardized incidence ratio in men 91 and in women 97). In the German Vegetarian Study, although the overall SMR for cancer was significantly reduced in men, there was no significant difference in risk in the strict vegetarians as compared to moderate vegetarians and no significant reduction in women (Chang-Claude et al. 1992).

An early report described the low death rate from various cancers amongst the Californian Adventist cohort (Phillips, 1975). Since the Adventists did not drink alcohol or smoke cigarettes, a reduction in risk of the cancers known to be related to either smoking or drinking is entirely expected. However, the SMR from cancer of the colon (61 in men and 70 in women) and postmenopausal cancer of the breast, ovary and uterus (70,53 and 60 respectively) were also significantly lower in the Adventist cohort.

Phillips went on to compare the rates of cancer in the Adventist cohort with those recorded in a large cohort of non-smokers, and showed that the rate of lung cancer in the 


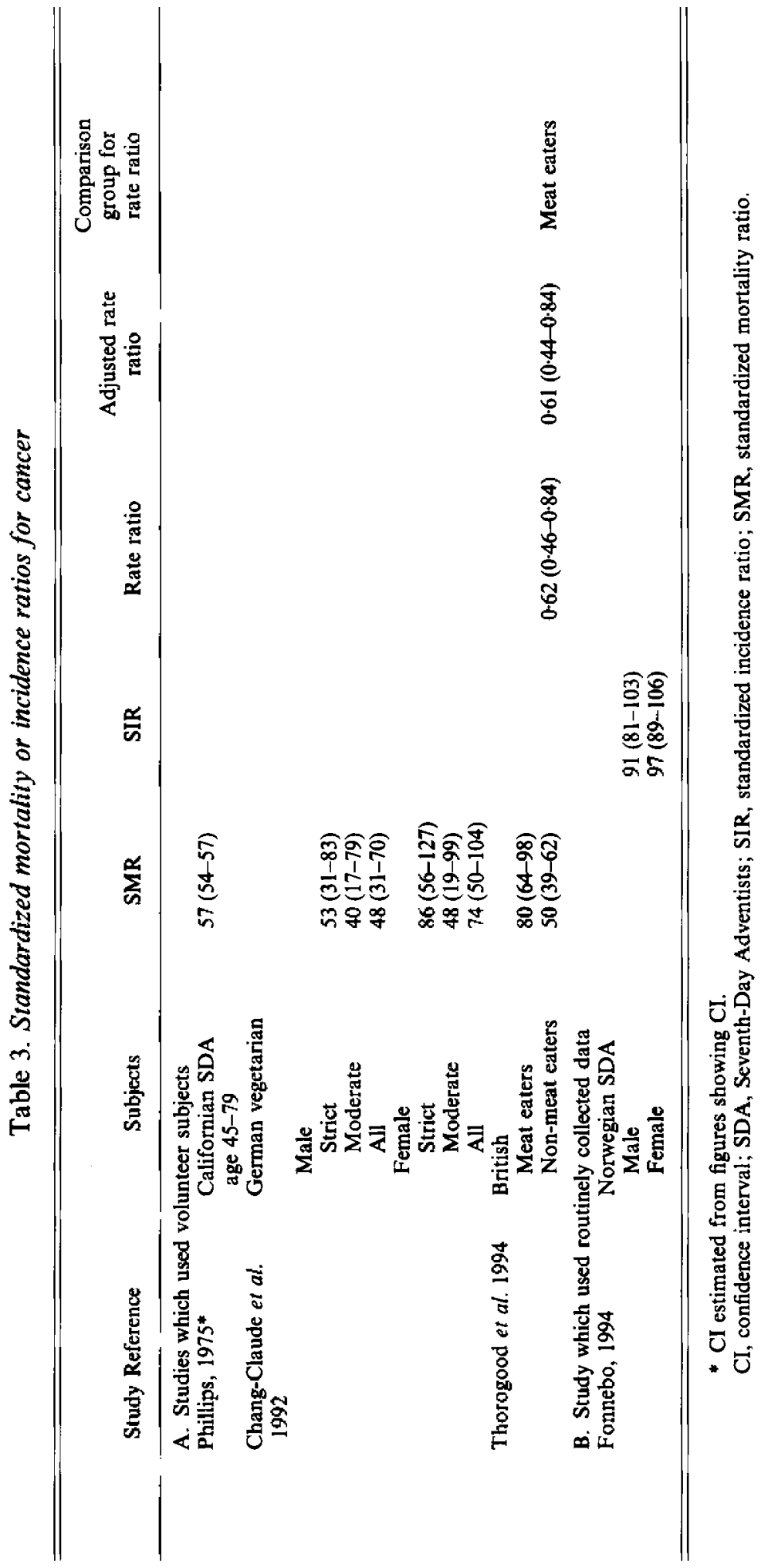


Adventist cohort was around $40 \%$ of that in the non-Adventist non-smokers--a difference which was statistically significant (Phillips, 1975). This analysis suggested that the Adventists may have experienced some protection from lung cancer which was additional to the very great protection of being non-smokers. A later analysis of data from the same cohort examined 61 new cases of lung cancer which occurred between 1977 and 1982 (Fraser et al. 1991). Although only $4 \%$ of the cohort were smokers, there was a twelve-fold increase in risk related to current cigarette smoking. The authors went on to examine the risk of lung cancer in relation to dietary variables and found no relationship between risk of lung cancer and reported meat consumption, or any other item of diet except fruit. There was a strongly protective effect of fruit, with subjects who ate fruit at least twice a day having about one quarter of the risk of those who ate fruit less than 3 times a week (relative risk $0 \cdot 26,95 \%$ CI $0 \cdot 10-0 \cdot 70$ ).

Data from the Californian Adventist study have also been analysed with respect to the relationship between reported intake of animal products (meat, eggs, cheese and milk) and risk of cancers of the breast, ovary, prostate and colon and rectum (Snowdon, 1988). Ovarian cancer mortality was found to be significantly related to egg consumption, with a threefold increase in risk among women who ate eggs on more than three days a week compared with those who never ate eggs $(P<0.01)$. There was also a suggestive but nonsignificant and smaller relationship with meat consumption. Prostate cancer was found to be related to consumption of a combination of four animal products (meat, eggs, cheese and milk), with a relative risk of 3.6 in high consumers as compared with low consumers (the author did not define high and low consumption nor did he provide a significance level for this observation). Colon cancer showed a moderate relationship with egg consumption only, while none of the four animal products was related to rectal or breast cancer.

The relationship between vegetarianism and breast cancer has also been examined in a case-control study of breast cancer carried out in Bombay (Rao et al. 1994a). Six hundred and eighty nine cases of breast cancer were interviewed, along with a control group of 711 women admitted to hospital for other causes. About $20 \%$ of the women were vegetarian, but no relationship was found between vegetarianism and risk of breast cancer.

A similar study by the same group examined the risk of male oral cancer associated with diet and found an $80 \%$ increase in risk associated with a non-vegetarian diet (Rao et al. $1994 \mathrm{~b}$ ). Again, around $20 \%$ of the men were vegetarian, and the age adjusted relative risk of oral cancer in the non-vegetarians as compared with the vegetarians was $1.83(95 \% \mathrm{CI}$ $1 \cdot 35-2 \cdot 5)$.

In general, the cohorts described here have low rates of cancer, but there is little evidence relating this to the absence of meat in the diet except, perhaps, for cancer of the prostate and mouth. The available evidence suggests that there is not a relationship between meat consumption and breast cancer. Future research (discussed below) may provide clearer information on this important topic.

\section{HYPERTENSION}

Some observational studies have shown lower blood pressure in vegetarians than in meat eaters. However, in a cross-sectional survey of some of the participants in the Health Food Shop Users study (Burr et al. 1981) there was no difference in blood pressure between the vegetarians and the meat eaters, suggesting that it may be aspects of vegetarianism which are shared in common with other health food users rather than the avoidance of meat which affect blood pressure.

There have been several clinical trials which have demonstrated that blood pressure can be lowered in subjects with both normal blood pressure and mild hypertension after 
changing to a vegetarian diet (Rouse et al. 1983; Margetts et al. 1986). Researchers conducting a recent trial randomized twenty fit non-smoking men to eat either an omnivorous or a vegetarian diet for a period of 6 weeks, during which their blood pressure was monitored with an ambulatory blood pressure monitoring device. Daytime systolic blood pressure was lowered in the subjects eating the vegetarian diet. After 6 weeks, the group on the vegetarian diet showed a significant fall in standing systolic blood pressure of $7.4 \mathrm{mmHg}$, compared with an increase in the omnivore group of $5.7 \mathrm{mmHg}$. A preprandial rise in diastolic blood pressure was observed in both groups, but this effect was attenuated in the vegetarian group (Sciarrone et al. $1993 a, b$ ). This trial did not attempt to disentangle the effects of different aspects of a vegetarian diet. One clinical trial randomized 60 volunteers to one of two diets which were identical except for the nature of the protein. One group ate a diet in which $40 \%$ of the protein came from meat, while the other group ate no meat. After 12 weeks there was no difference in blood pressure between the two groups (Prescott et al. 1988). This was a small trial of short duration, and therefore had limited power to detect real but small differences between the groups, but it is at least suggestive of the possibility that the blood pressure lowering effect of a vegetarian diet is not due to the absence of meat in the diet.

The relationship between vegetarianism and blood pressure has recently been reviewed by Beilin (1993) who considered 23 papers. He concluded that attempts to identify which of many nutrient differences in a vegetarian diet might account for the lower blood pressure in vegetarians had been inconclusive. Differences in potassium and sodium intake or meat intake do not appear to account for the difference, and trials of fat reduction or fibre increase have been inconsistent.

\section{BLOOD LIPID LEVELS}

Total, low density lipoprotein (LDL) cholesterol, and high density lipoprotein (HDL) cholesterol were measured in some 3200 subjects from the Oxford Vegetarian Study. Both total and LDL cholesterol were significantly lower in subjects who did not eat meat, with the lowest values being observed in vegans (who did not eat any animal products at all). HDL cholesterol was not affected by diet, except for a very small, but statistically significant, rise in the group of people who did not eat meat, but did eat fish (Thorogood et al. 1987). The reduction of total and LDL cholesterol among vegetarians has been observed in other studies in both the UK and the USA (Sacks et al. 1975; Burr et al. 1981).

It is interesting that the same relationship between vegetarians and meat eaters still applies when the diet of the meat eating population is substantially lower in fat than the average diet of a western country. A study in Thailand compared the nutrient intake and blood lipid levels of 132 Buddhist vegetarians with those of 68 similar meat eaters, who were consuming a very low fat diet by western standards, with around $20 \%$ of their energy being derived from fat. Nevertheless, the fat content of the vegetarian diet was lower, at around $12 \%$ of energy, and total, LDL, and HDL cholesterol concentrations were significantly lower. The mean total cholesterol levels in the vegetarian men and women were $4.0 \mathrm{mmol} / 1(95 \% \mathrm{CI} 3.8-4.4)$ and $4.3 \mathrm{mmol} / 1$ (95\% CI $4.2-4.4)$ respectively, while in the non-vegetarians the equivalent levels were $5 \cdot 3 \mathrm{mmol} / 1(95 \% \mathrm{CI} 5 \cdot 0-5 \cdot 6)$ and $5 \cdot 3(95 \% \mathrm{CI}$ 4.9-8.4) respectively (Supawan et al. 1992).

There is some evidence that the relationship between blood cholesterol and vegetarian diet is not uniform. Reddy \& Sanders (1992) compared the lipoprotein concentrations of 22 vegetarian women of Indian descent living in southern England with those of 18 similar white vegetarians and 22 meat eaters. The proportion of energy derived from polyunsaturated fatty acids was significantly greater in both groups of vegetarians than in 
the omnivores $(7.8,7.6$ and $6.8 \%$ respectively in white vegetarians, Indian vegetarians, and white omnivores), and the proportion of energy derived from saturated fatty acids was significantly lower $(11.4,11.4$ and $15.7 \%$ respectively). However, while the total cholesterol level was significantly lower in white vegetarians than in meat eaters $(4.44 \mathrm{mmol} / \mathrm{l}$ and $5.19 \mathrm{mmol} / 1$ respectively), the total cholesterol level in the Indian vegetarians (4.78 mmol/1) was not significantly different from that of the meat eaters. Plasma concentrations of LDL cholesterol were lower, and concentrations of $\mathrm{HDL}$ and $\mathrm{HDL}_{2}$ were significantly greater in the white vegetarian group than in the other two groups. Total plasma cholesterol concentration was significantly associated with measures of truncal obesity, particularly subscapular skinfold thickness, which was significantly greater in the Indian vegetarians than in the other two groups.

\section{DIABETES}

Snowdon \& Phillips (1985) examined the relationship between vegetarianism and the risk of diabetes within the Californian Adventist cohort. After allowing for the confounding effects of differences between vegetarians and non-vegetarians in ideal body weight and reported physical activity there was still a significant increase in the likelihood of diabetes being mentioned on the death certificate amongst the non-vegetarian men, with men who ate meat six or more times a week being 3.6 times more likely to have such a mention on the death certificate $(95 \%$ CI 1.9-7.1). No such relationship was observed in the women.

In a small non-randomized cross-over study of dietary therapy in eight patients with diabetic neuropathy Jibani and colleagues (1991) have demonstrated a significant reduction in albumin excretion rate during a period on a vegetarian diet. Since the number of subjects was so small, and the trial was not randomized, it is not possible to draw any useful conclusions except that such an observation is potentially important and should be explored further in a randomized controlled trial.

\section{CORONARY HEART DISEASE}

Some of the important modifiable risk factors for coronary heart disease, that is, raised blood pressure and an unfavourable serum lipid profile, have been shown to be connected with a vegetarian diet, as well as a possible relationship between diabetes and vegetarianism. In the light of these relationships, it is not surprising that many of the cohort studies have observed an inverse relationship between coronary heart disease mortality and vegetarianism. The findings of the cohort studies in relation to coronary heart disease are summarized in Table 4.

Mortality from ischaemic heart disease was studied in the Health Food Shop Users Study. Both vegetarians and non-vegetarians had low rates of fatal ischaemic heart disease compared with the general population. The SMR for ischaemic heart disease was 60 in nonvegetarians, but was significantly lower at 43 in vegetarians (Burr \& Butland, 1988). In the German Vegetarian Study (Chang-Claude \& Frentzel-Beyme, 1993) the rate of fatal ischaemic heart disease was about half as low among the strict vegetarians as among the moderate vegetarians, although this difference did not quite achieve significance (relative risk $0.52,95 \%$ CI $0.95-1 \cdot 05$ ).

In the Oxford Vegetarian Study both the meat eaters and the non-meat eaters had a low death rate from ischaemic heart disease in comparison with the population of England and Wales. The SMR for ischaemic heart disease was 51 (95\% CI 38-66) in meat eaters but significantly lower at $28(95 \%$ CI $20-38)$ in non-meat eaters. However, after adjustment had been made for the differences in body mass index, smoking history and social class, the 
Table 4. Standardized mortality ratios and rate ratios for ischaemic heart disease mortality

\begin{tabular}{|c|c|c|c|c|c|}
\hline Study Reference & Subjects & SMR & Rate ratio & Relative risk & Comparison GP \\
\hline \multirow[t]{5}{*}{ Phillips et al. 1978} & $\begin{array}{l}\text { Californian SDA } \\
\text { All males age }\end{array}$ & $26(18-36)$ & & & \\
\hline & $\begin{array}{l}\text { 35-64 } \\
\text { As above - }\end{array}$ & & $3 \cdot 1(1 \cdot 1-7 \cdot 9)$ & & Vegetarian \\
\hline & Non-vegetarian only & & & & \\
\hline & $\begin{array}{l}\text { All female age } \\
35-64\end{array}$ & $34(21-53)$ & & & \\
\hline & $\begin{array}{l}\text { As above - } \\
\text { Non-vegetarian only }\end{array}$ & & $1 \cdot 2(0 \cdot 4-2 \cdot 7)$ & & Vegetarian \\
\hline \multirow[t]{4}{*}{ Snowdon et al. 1984} & Californian SDA & & & & \\
\hline & $\begin{array}{l}\text { Non-vegetarian age } \\
4584 \text { : }\end{array}$ & & & & \\
\hline & Male & & & $1.5(1.3-1.7)^{*}$ & Vegetarian \\
\hline & Female & & & $1.4(1.2-1 \cdot 6)^{*}$ & Vegetarian \\
\hline \multirow{4}{*}{$\begin{array}{l}\text { Chang-Claude et al. } \\
1992\end{array}$} & German vegetarians & & & & \\
\hline & Male & & & & \\
\hline & Strict & $27(12-50)$ & & & \\
\hline & Moderate & $53(25-97)$ & & & \\
\hline \multirow{3}{*}{$\begin{array}{l}\text { Chang-Claud \& } \\
\text { Frentzel-Beyme, } 1993\end{array}$} & Female & & & & \\
\hline & $\begin{array}{l}\text { Strict } \\
\text { Moderate }\end{array}$ & $\begin{array}{l}24(9-52) \\
47(15-108)\end{array}$ & & & \\
\hline & All moderate & & & $1.9(0.95-4.0)$ & Strict \\
\hline \multirow[t]{4}{*}{ Burr \& Butland, 1988} & British Health & & & & \\
\hline & Food Shop Users & & & & \\
\hline & Vegetarian & 43 & & & \\
\hline & Non-vegetarian & & & & \\
\hline \multirow{2}{*}{ Thorogood et al. 1994} & British meat eaters & $51(38-66)$ & & & \\
\hline & Non-meat eaters & $28(20-38)$ & $0.7(0.5-1.1) \dagger$ & & \\
\hline
\end{tabular}

* Age adjusted.

+ Adjusted for age, sex, social class, smoking history and body mass index.

GP, group; SDA, Seventh-Day Adventists; SMR, standardized mortality ratio. All studies used volunteer subjects.

resulting $25 \%$ reduction in death from ischaemic heart disease among non-meat eaters as compared with meat eaters was no longer significant. The rate ratio for non-meat eaters as compared with meat eaters was $0.72(95 \%$ CI 0.47-1.10) (Thorogood et al. 1994).

It is the Californian Adventist Study which provides the best evidence of a possible effect of meat consumption on coronary heart disease. A study of fatal ischaemic heart disease within the Californian Adventist cohort compared the rate of events in vegetarians and non-vegetarians after 20 years of follow-up and showed about a $50 \%$ increase in risk in those who ate meat (Snowdon et al. 1984). The age adjusted relative risk in meat eaters as compared with vegetarians was $1.5(95 \% \mathrm{CI} 1.3-1.7)$ in men and $1.4(95 \% \mathrm{CI} 1.2-1.6)$ in women. Moreover, there was clear evidence of a dose response, in that there was a significant trend of an increasing risk as the frequency of meat consumption increased.

A recent re-analysis of the Californian Adventist data confirmed the importance of meat consumption as being associated with around a $50 \%$ significant increase in the risk of fatal ischaemic heart disease in Adventists who ate beef more than twice a week. The same analysis, however, showed an important effect of nut consumption. The risk of fatal ischaemic heart disease in Adventists who ate nuts more than four times a week was just 
over half that in Adventists who never ate nuts (relative risk 0.59, 95\% CI 0.45-0.78). This relationship held after beef consumption was taken into account. In other words, regular eating of nuts was associated with a reduced risk of fatal ischaemic heart disease in both vegetarians and non-vegetarians (Fraser et al. 1992).

\section{OTHER DISEASES}

A relationship between vegetarianism and a variety of other diseases has been observed. The onset of dementia in meat eaters and non-meat eaters has been studied in the Californian Adventist cohort (Giem et al. 1993). The analysis was done two ways: in one case 63 vegans age over 65 were matched with 63 similar vegetarians and 63 heavy meat eaters (defined as eating meat at least 5 times a week); in the other case the onset of dementia was observed in an unmatched analysis of 2984 subjects from the original Adventist cohort. These two analyses produced different results. In the matched analysis of the smaller group there appeared to be a significant three-fold increase in risk of dementia in heavy meat eaters compared to those who had not eaten meat for 30 years, but in the unmatched analysis there was no significant difference between the various dietary groups. The results, then, were inconclusive but intriguing. In a rapidly ageing population, investigation of possible risk factors for dementia are of major public health importance. Further research in this area would be of great interest.

Another issue of particular importance in old age is that of bone density. In a cohort study of a small group of vegetarian and meat eating elderly women, researchers measured bone loss over a period of 5 years. The study was bedevilled by losing more than half of the original cohort, mainly due to death or failing health. However, among the 189 women who were examined at the beginning and end of the study, there was no difference in bone loss between the vegetarians and the meat eaters, with both groups having lost bone mass in the five year period at the same rate (Reed et al. 1994).

\section{THE FUTURE}

The data which are available from the existing cohort studies of vegetarians have proved fascinating, but also inconclusive. The indication that something about a vegetarian life is related to a considerable health advantage is strong, but the limitations of the studies in terms of size and of the comprehensiveness of the available data lead to the frustration of being unable to disentangle whether, and to what extent, the consumption of meat has a causative role in this relationship. The role of diet in the aetiology of disease is unlikely to be elucidated by clinical trials, which are inevitably limited in size and duration. Casecontrol studies will also have limited value, since the information on diet is, perforce, collected retrospectively once an illness is already established and after a diagnosis has been made. What is needed, then, is large cohort studies in which detailed data on potential confounding factors and on dietary intake are carefully collected prospectively. The European Prospective Study into Cancer and Nutrition (the EPIC Study) is just such a study. The EPIC team plan to collect data on some 400000 adult subjects in seven European countries: UK, The Netherlands, Germany, France, Italy, Spain and Greece (Riboli, 1992). The subjects will be followed up to investigate both mortality and cancer incidence. One of the strengths of the study will therefore be the range of very heterogeneous dietary intakes. Another strength will be that blood samples are being collected and frozen in small aliquots so that biochemical markers of dietary intake will also be available to the researchers. It is particularly encouraging that one of the UK 
collaborating centres, the Imperial Cancer Research Fund Cancer Epidemiology Unit, is making a special effort to include a very large subcohort of vegetarians in the study.

\section{SUMMARY AND CONCLUSION}

The vegetarian subjects in the studies described in this paper appear on the whole to have enjoyed a better standard of health than have the groups they are compared with. Some, at least, of this health advantage may be explained by various factors not related to diet, such as the 'healthy volunteer effect', the low smoking prevalence, or the high social class of vegetarians. In other cases, notably some forms of cancer, hypertension, and coronary heart disease, the effects seem to be more clearly related to diet. It is not possible to be certain whether this is due to a higher intake of some foods in the vegetarian diet, or to the absence of others. Dietary intakes are so closely inter-related that it is often impossible to determine whether the important factor is the abundance of fruit and vegetables, the avoidance of meat, or the high P:S ratio, or, indeed, the regular consumption of nuts which is most important.

The current multinational EPIC study will have sufficient power to disentangle some of these effects, but the very close interaction between the different constituents of a normal diet may mean that some of the effects are never fully teased out. What is clear is that a diet high in fruit and vegetables and low in saturated fatty acids brings with it substantial health benefits. An apple a day (along with one or two other things) does indeed keep the doctor away.

\section{REFERENCES}

Beilin, L. J. (1993). Vegetarian diets, alcohol consumption, and hypertension. Annals of the New York Academy of Sciences 676, 83-91.

Burr, M. L., Bates, C. J., Fehily, A. M. \& St Leger, A. S. (1981). Plasma cholesterol and blood pressure in vegetarians. Journal of Human Nutrition 35, 437-441.

Burr M. L. \& Butland, B. K. (1988). Heart disease in British vegetarians. American Journal of Clinical Nutrition 48, 830-832.

Chang-Claude, J. \& Frentzel-Beyme, R. (1993). Dietary and lifestyle determinants of mortality among German vegetarians. International Journal of Epidemiology 22, 228-236.

Chang-Claude, J., Frentzel-Beyme, R. \& Eilber, U. (1993). Mortality pattern of German vegetarians after 11 years of follow-up. Epidemiology 3, 395-401.

Diplock, A. T. (1991). Antioxidant nutrients and disease prevention: an overview. American Journal of Clinical Nutrition 53, 189S-193S.

Dwyer, J. T. (1991). Nutritional consequences of vegetarianism. Annual Review of Nutrition 11, 61-91.

Fonnebo, V. (1994). The healthy Seventh-day Adventist lifestyle: what is the Norwegian experience? American Journal of Clinical Nutrition 59, 1124S-1129S.

Fraser, G. E., Beeson, W. L. \& Phillips, R. L. (1991). Diet and lung cancer in California Seventh-day Adventists. American Journal of Epidemiology 133, 683-93.

Fraser, G. E., Sabate, J., Beeson, W. L. \& Strahan, T. M. (1992). A possible protective effect of nut consumption on risk of coronary heart disease: the Adventist Health Study. Archives of Internal Medicine 152, 1416-1428.

Giem, P., Beeson, W. L. \& Fraser, G. E. (1993). The incidence of dementia and intake of animal products: preliminary findings from the Adventist Health Study. Neuroepidemiology 12, 28-36.

Jibani, M. M., Bloodworth, L. L., Foden, E., Griffiths, K. \& Galpin, O. P. (1991). Predominantly vegetarian diet in patients with incipient and early clinical diabetic nephropathy: effects on albumin excretion rate and nutritional status. Diabetic Medicine 8, 949-953.

Knutsen, S. F. (1994). Lifestyle and the use of health services. American Journal of Clinical Nutrition 59, $1171 \mathrm{~S}-1175 \mathrm{~S}$.

McMichael, A. J. (1992). Vegetarians and longevity: imagining a wider reference population. Epidemiology 3, 389-391.

Margetts, B. M., Beilin, L. J., Vandongen, R. \& Armstrong. B. K. (1986). Vegetarian diet in mild hypertension: a randomised controlled trial. British Medical Journal 293, 1468-1471.

Phillips, R. L. (1975). Role of lifestyle and dietary habits in risk of cancer among Seventh-Day Adventists. Cancer Research 35, 3513-3522. 
Phillips, R. L., Garfinkel, L., Kuzma, J. W., Beeson, W. L., Lotz, T. \& Brin, B. (1980). Mortality among California Seventh-Day Adventists for selected cancer sites. Journal of the National Cancer Institute 65, 1097-1107.

Phillips, R. L., Lemon, F. R., Beeson, W. L. \& Kuzma, J. W. (1978). Coronary heart disease mortality among Seventh-Day Adventists with differing dietary habits: a preliminary report. American Journal of Clinical Nutrition 31 (10, Suppl.), S191-S198.

Prescott, S. L., Jenner, D. A., Beilin, L. J., Margetts, B. M. \& Vandongen, R. (1988). A randomized controlled trial of the effect on blood pressure of dietary non-meat protein versus meat protein in normotensive omnivores. Clinical Science 74, 665-672.

Rao, D. N., Ganesh, B. \& Desai, P. B. (1994a). Role of reproductive risk factors in breast cancer in a low-risk area: a case-control study. British Journal of Cancer 70, 129-132.

Rao, D. N., Ganesh, B., Rao, R. S. \& Desai, P. B. (1994b). Risk assessment of tobacco, alcohol and diet in oral cancer-a case-control study. International Journal of Cancer 58, 469-473.

Realeat Company (1984). The 1984 REALEAT Meat-eating Survey. London: Realeat Co.

Realeat Company (1993). The 1993 REALEAT Meat-eating Survey. London: Realeat Co.

Reddy, S. \& Sanders, T. A. B. (1992). Lipoprotein risk factors in vegetarian women of Indian descent are unrelated to dietary intake. Atherosclerosis $95,223-229$.

Reed, J. A., Anderson, J. J., Tylavsky, F. A. \& Gallagher, P. N. (1994). Comparative changes in radial-bone density of elderly female lacto-ovovegetarians and omnivores. American Journal of Clinical Nutrition 59 , $1197 \mathrm{~S}-1202 \mathrm{~S}$.

Riboli, E. (1992). Nutrition and cancer: background and rationale of the European Prospective Investigation into Cancer and Nutrition (EPIC). Annals of Oncology 3, 783-791.

Rouse, I. L., Amstrong, B. K. \& Beilin, L. J. (1983). The relationship of blood pressure to diet and lifestyle in two religious populations. Journal of Hypertension 1, 65-71.

Sacks, F. M., Castelli, W. P., Donner, A. \& Kass, E. H. (1975). Plasma lipids and lipoproteins in vegetarians and controls. New England Journal of Medicine 292, 1148-1151.

Sciarrone, S. E. G., Strahan, M. T., Beilin, L. J., Burke, V., Rogers, P. \& Rouse, I. (1993a). Ambulatory blood pressure and heart rate responses to vegetarian meals. Journal of Hypertension 11, 277-285.

Sciarrone, S. E. G., Strahan, M. T., Beilin, L. J., Burke, V., Rogers, P. \& Rouse, I. (1993b). Biochemical and neurohormonal responses to the introduction of a lacto-ovovegetarian diet. Journal of Hypertension 11, 849-860.

Snowdon, D. A. (1988). Animal product consumption and mortality because of all causes combined, coronary heart disease, stroke, diabetes, and cancer in Seventh-day Adventists. American Journal of Clinical Nutrition 58, 739-748.

Snowdon, D. A. \& Phillips, R. L. (1985). Does a vegetarian diet reduce the occurrence of diabetes? American Journal of Public Health 75, 507-512..

Snowdown, D. A., Phillips, R. L. \& Fraser, G. E. (1984). Meat consumption and fatal ischaemic heart disease. Preventive Medicine 13, 490-500.

Supawan, V., Pongpaew, T., Tungtrongchitr, R., Tawprasert, S., Changbumrung, S., Migasena, P. \& Schelp, F. P. (1992). Lipid profile, anthropometry and dietary intake of 132 Thai vegetarians. International Journal for Vitamin and Nutrition Research 62, 324-329.

Thorogood, M., Carter, R., Benfield, L., McPherson, K. \& Mann, J. I. (1987). Plasma lipids and lipoprotein cholesterol concentrations in people with different diets in Britain. British Medical Journal 295, $351-353$.

Thorogood, M., Mann, J., Appleby, P. \& McPherson, K. (1994). Risk of death from cancer and ischaemic heart disease in meat-eaters and non-meat eaters. British Medical Journal 308, 1667-1671.

Vegetarian Society (1991). Trends in Vegetarianism among Adults and Young People. Altrincham: The Vegetarian Society (UK) Ltd. 